\title{
Indications and Outcomes for Pedicled Pectoralis Major Myocutaneous Flaps at a Primary Microvascular Head and Neck Reconstructive Center
}

\author{
Akira Saito $^{1}$, Hidehiko Minakawa ${ }^{1}$, Noriko Saito ${ }^{2}$, Tatsumi Nagahashi ${ }^{3}$ \\ ${ }^{1}$ Division of Plastic and Reconstructive Surgery, Hokkaido Cancer Center, Sapporo, Japan; ${ }^{2}$ Department of Plastic and Reconstruc- \\ tive Surgery, Graduate School of Medicine, Hokkaido University, Sapporo, Japan; ${ }^{3}$ Division of Head and Neck Surgery, Hokkaido \\ Cancer Center, Sapporo, Japan. \\ Email: rogoredo84@gmail.com
}

Received July $14^{\text {th }}, 2012$; revised August $16^{\text {th }}, 2012$; accepted September $17^{\text {th }}, 2012$

\begin{abstract}
The pectoralis major musculocutaneous (PMMC) flap has been a useful technique for head and neck reconstruction since its first description by Ariyan in 1979. However, techniques in microvascular surgery have since evolved and recently free tissue transfer has played an important role in head and neck reconstruction. Although we use free flaps as the first choice for head and neck reconstruction, similar to many other institutions, some patients at our hospital have undergone reconstruction with PMMC flaps. We retrospectively analyzed the indications and outcomes of this reconstructive technique from our experience with 12 patients. The medical records of all patients who underwent PMMC flaps at Hokkaido Cancer Center from 2001 to 2010 were reviewed. Data concerning diagnosis, main indication, site of reconstruction, previous treatment, and postoperative complications were analyzed. Of the 12 PMMC flap surgeries performed, 3 were carried out as primary reconstructive procedures, whereas 9 were done as "salvage" procedures. Flap-related complications were observed in 6 cases. Partial flap loss developed in 4 patients, although there were no cases of total flap loss. There were 3 recurrent fistulae following reconstruction with PMMC flaps. The preoperative goals of performing PMMC flap surgery were met in $83 \%$ of our cases. The authors conclude that while free flap transfer is usually the first choice for head and neck reconstruction, PMMC flaps can produce acceptable results in certain situations.
\end{abstract}

Keywords: Pectoralis Major Myocutaneous Flap; Head and Neck Reconstruction; Pedicled Flap; Pedicled Pectoralis Major Myocutaneous Flap; Reconstruction, Microsurgery

\section{Introduction}

Since its first description by Ariyan in 1979 [1], pectoralis major musculocutaneous (PMMC) flap surgery has been a useful technique for head and neck reconstruction [2-5], the advantages of which include robust size, versatility, and determinate blood supply [6]. However, since the early 1980s, techniques of microvascular surgery have evolved and free tissue transfer has played an important role in reconstructive surgery for advanced head and neck cancers [7].

There have been studies comparing the differences between free flaps and pedicled myocutaneous flaps for head and neck reconstruction which suggest that free flaps are superior with regard to postoperative results and cost effectiveness [8,9]. Currently, free tissue transfer is the first choice for head and neck reconstruction at many institutes [10].
However, free flap transfer cannot be used for all patients at every institute since it requires special techniques and equipment for microsurgery, and it takes a longer time to perform than does the pedicled myocutaneous flap. Although we usually use free flaps as the first choice for head and neck reconstruction, some patients at our institution have undergone reconstruction using PMMC flaps. We retrospectively analyzed the indications and outcomes for this technique from our experience with 12 patients.

\section{Methods}

The medical records of all patients who underwent PMMC flap surgery at Hokkaido Cancer Center from 2001 to 2010 were reviewed. Thirteen patients were identified as having undergone a PMMC flap for head and neck reconstruction, but 1 patient was excluded due 
to a lack of adequate information. The medical charts of the 12 participating patients were retrospectively reviewed for data regarding diagnosis, main indication, site of reconstruction, previous treatment, and postoperative complications. Major complications were defined as those requiring revision surgeries, while minor complications required conservative wound care alone. Statistical analyses were performed by Fisher's exact probability tests. A value of $p<0.05$ was considered statistically significant.

\section{Results}

Patient demographics and prior treatments are presented in Table 1. There were 11 males and 1 female patient, with a mean age of 66.3 years (range: 27 - 83 years).

The distribution of cases regarding site of reconstruction, indication for PMMC flaps, type of reconstruction, and the requirement for skin grafts is shown in Table 2. Of the 12 PMMC flaps, 3 were carried out as primary reconstructive procedures, whereas 9 were done as "salvage" procedures ( 5 for fistula, 2 for free flap failure, 1 for ablation of recurrent tumor after a free flap reconstruction, and 1 for ablation of recurrent tumor after radiation therapy). A titanium reconstruction plate was used to restore mandibular continuity in conjunction with

Table 1. Patient and characteristics.

\begin{tabular}{lc}
\hline \multicolumn{1}{c}{ Characteristics } & $\mathrm{n}$ \\
\hline Patients & 12 \\
Male & 11 \\
Female & 1 \\
Age, mean (range), years & $66.3(27-83)$ \\
Disease & \\
Laryngeal cancer & 4 \\
Floor of mouth cancer & 3 \\
Tongue cancer & 2 \\
Hypopharyngeal cancer & 1 \\
Esophageal cancer & 1 \\
Skin cancer & 1 \\
Prior treatment & \\
Surgery only & \\
Surgery + RT & 3 \\
Surgery + CCRT & 4 \\
CCRT & 2 \\
None & \\
\hline
\end{tabular}

Table 2. Case distribution in relation to reconstructive surgery.

\begin{tabular}{|c|c|}
\hline Site of reconstruction & $\mathrm{n}$ \\
\hline Neck & 6 \\
\hline Oral cavity & 5 \\
\hline Cheek & 1 \\
\hline \multicolumn{2}{|l|}{ Indication for PMMC flap } \\
\hline Repair of fistula & 5 \\
\hline Pharyngocutaneous fistula & 4 \\
\hline Gastric tube-skin fistula & 1 \\
\hline Repair of defect following tumor ablation & 5 \\
\hline Primary tumor & 3 \\
\hline Recurrent tumor & 2 \\
\hline Repair of total flap loss & 2 \\
\hline \multicolumn{2}{|l|}{ Type of reconstruction } \\
\hline PMMC flap & 11 \\
\hline PMMC flap + reconstruction plate & 1 \\
\hline \multicolumn{2}{|l|}{ Skin graft } \\
\hline Yes & 4 \\
\hline Donor site & 1 \\
\hline On the muscle & 3 \\
\hline No & 8 \\
\hline
\end{tabular}

the PMMC flap.

Flap-related complications were observed in 6 cases (50\%) and are shown in Table 3. Major complications were observed in 3 patients (25\%) and minor complications were seen in 3 patients (25\%). Partial flap loss developed in 4 patients, although there were no cases of total flap loss. There were 3 recurrent fistulae following reconstruction with PMMC flaps.

Data regarding comparisons between the occurrence of complications and indications or previous treatments are presented in Table 4. Among the 7 patients who received prior radiotherapy, 5 (71\%) developed complications, whereas only 1 patient (20\%) developed complications among the 5 who did not receive prior radiotherapy.

\section{Discussion}

Free flap transfers have become the first choice for head and neck reconstruction surgeries at many institutions. This procedure provides a one-stage restoration with significantly lower morbidity and complication rate at donor and recipient sites, and usually has better outcomes than 
Table 3. Complications following reconstructive surgery with PMMC flaps.

\begin{tabular}{ll}
\hline Complications & $\mathrm{n}$ \\
\hline Yes & 6 \\
Major complications & 3 \\
Minor complications & 3 \\
No & 6 \\
Detail of complications & \\
Partial flap necrosis & 4 \\
Fistula & 4 \\
Wound dehiscence & 1 \\
\hline
\end{tabular}

Table 4. Comparisons between the presence of complication and variables of interest.

\begin{tabular}{|c|c|c|}
\hline \multirow{2}{*}{ Variables } & \multicolumn{2}{|c|}{ Complication } \\
\hline & Yes & No \\
\hline \multicolumn{3}{|l|}{ Indication } \\
\hline Fistula & 4 & 1 \\
\hline Others & 2 & 5 \\
\hline \multicolumn{3}{|l|}{ Previous RT therapy } \\
\hline Yes & 5 & 2 \\
\hline No & 1 & 4 \\
\hline \multicolumn{3}{|l|}{ Previous surgery } \\
\hline Yes & 5 & 4 \\
\hline No & 1 & 2 \\
\hline
\end{tabular}

alternative approaches [5,11]. At out institute, we also use free flaps as the first choice for head and neck reconstruction, including free anterolateral thigh flaps, free radial forearm flaps, free rectus abdominis flaps, and free jejunum transfer. Nowadays, free flaps are more common due to improved microsurgical techniques, while PMMC flaps have lost much of their reputation for reconstructtion of the head and neck region [12].

However, PMMC flaps still have a place in head and neck reconstruction. This technique can be used as a salvage procedure after necrosis of free flaps and in cases where there are contraindications to free flaps, such as medical conditions that make the patient unable to tolerate long surgical procedures or inadequate recipient vessels for microanastomosis in the necks of patients who previously underwent high-dose radiotherapy [5]. PMMC flaps can also be performed in combination with free flaps, usually for covering large defects, to protect neck vessels in patients that are at risk for rupture, and to prevent possible complications of wounds that have a high risk of breakdown [13].

Schneider et al. have previously described the indications for PMMC flap surgery at a primary microvascular head and neck reconstructive center. In their series of 53 patients, PMMC flaps were used: 1 ) to salvage free flap complications (38\%), 2) with simultaneous free flap reconstruction for additional soft tissue filler in extensive resections, or for cervical skin reconstruction (33\%), and 3) for primary reconstructions, most frequently involving compromised host status with a need for cervical skin reconstruction and great vessel coverage after radical neck dissection (29\%) [14]. In our series, primary reconstruction with PMMC flaps was performed on 3 patients (25\%), while the other 9 patients (75\%) underwent "salvage" reconstructions (i.e., reconstruction after free flap failure, fistulas, and recurrent tumor ablation). Since 2 of them were elderly and another suffered from malnutrition, the 3 patients who underwent primary reconstruction with PMMC flaps were considered unable to tolerate long, invasive surgeries.

Flap-related complications developed in 6 (50\%) of the patients, with 3 experiencing major complications. Thus, the results of our series were comparable to previous reports, since overall complication rates of PMMC flaps have been reported to be quite high, ranging from $16 \%$ to $63 \%$ [5].

One of the main advantages of PMMC flaps is survival. Even if performed by an experienced microsurgeon, total flap loss can occur in free flap reconstructions. On the other hand, total loss of PMMC flaps is rare, although partial flap loss can occur [13]. In the current series, partial flap necrosis occurred in 4 patients (33\%), although all flaps survived. We suppose that it might occur since the skin paddle was designed more inferiorly beyond vascular territories for the flap in some cases. Two patients required revision surgery with other flaps because they developed fistulas following reconstruction with PMMC flaps. In all other patients, reconstructions with primary PMMC flaps were successful, with the reconstruction success rate being $83 \%$. These results are slightly lower than success rates of other studies, which ranged from $87.5 \%$ to $100 \%$ [13,15-17].

Previous reports have described the risk factors associated with the development of flap complications, such as age, sex, tumor location, site of reconstruction, prior radiotherapy, and comorbidities, large tumor resections, cigarette packs smoked, and salvage procedures $[3,15,18$, 19]. However, results were not similar in all series with some reports describing that complication rates were not associated with age, sex, smoking, preoperative radiotherapy, diabetes, or obesity [2,4,5,12]. 
In our series, 5 out of the 6 patients (83\%) who developed complications had a history of preoperative radiotherapy, while only 1 of 6 patients (17\%) who did not develop complications had undergone preoperative radiotherapy. However, this difference was not statistically significant.

Three out of 5 patients with fistula had recurrences after the reconstructive procedures, with 2 of them developing fistula recurrence following partial flap loss. Since many cases with fistula have a prior history of radiation, which delays healing, when the primary indication of the procedure is to repair a fistula, special care should be taken to safely elevate the flap in order to minimize necrosis.

In conclusion, of 12 PMMC flap surgeries performed at our institution, 3 were carried out as primary reconstructive procedures, whereas 9 were done as "salvage" procedures. Major complications were observed in 3 patients (25\%), and minor complications were seen in 3 patients (25\%). Partial flap loss developed in 4 patients (33\%), although there were no cases of total flap loss. The preoperative goals of the flaps were met in $83 \%$ of our cases.

\section{Conclusion}

The authors conclude that although free flap transfer is most often the first choice for head and neck reconstructtion, PMMC flaps can produce acceptable results in certain situations.

\section{REFERENCES}

[1] S. Ariyan, "The Pectoralis Major Myocutaneous Flap. A Versatile Flap for Reconstruction in the Head and Neck," Plastic and Reconstructive Surgery, Vol. 63, No. 1, 1979, pp. 73-81. doi:10.1097/00006534-197901000-00012

[2] I. J. CB, S. E. Hovius, B. L. ten Have, S. J. Wijthoff, G. J. Sonneveld, C. A. Meeuwis and P. P. Knegt, "Is the Pectoralis Myocutaneous Flap in Intraoral and Oropharyngeal Reconstruction Outdated?” The American Journal of Surgery, Vol. 172, No. 3, 1996, pp. 259-262. doi:10.1016/S0002-9610(96)00161-4

[3] S. Mehta, S. Sarkar, N. Kavarana, H. Bhathena and A. Mehta, "Complications of the Pectoralis Major Myocutaneous Flap in the Oral Cavity: A Prospective Evaluation of 220 Cases," Plastic and Reconstructive Surgery, Vol. 98, No. 1, 1996, pp. 31-37. doi:10.1097/00006534-199607000-00006

[4] R. Liu, P. Gullane, D. Brown and J. Irish, "Pectoralis major Myocutaneous Pedicled Flap in Head and Neck Reconstruction: Retrospective Review of Indications and Results in 244 Consecutive Cases at the Toronto General Hospital,” Journal of Otolaryngology, Vol. 30, No. 1, 2001, pp. 34-40. doi:10.2310/7070.2001.21011

[5] J. G. Vartanian, A. L. Carvalho, S. M. Carvalho, L.
Mizobe, J. Magrin and L. P. Kowalski, "Pectoralis Major and Other Myofascial/Myocutaneous Flaps in Head and Neck Cancer Reconstruction: Experience with 437 Cases at a Single Institution,” Head \& Neck, Vol. 26, No. 12, 2004, pp. 1018-1023. doi:10.1002/hed.20101

[6] A. L. McCrory and J. S. Magnuson, "Free Tissue Transfer versus Pedicled Flap in Head and Neck Reconstruction.” Laryngoscope, Vol. 112, No. 12, 2002, pp. 21612165. doi:10.1097/00005537-200212000-00006

[7] K. E. Blackwell, D. Buchbinder, H. F. Biller and M. L. Urken, "Reconstruction of Massive Defects in the Head and Neck: The Role of Simultaneous Distant and Regional Flaps," Head \& Neck, Vol. 19, No. 7, 1997, pp. 620-628.

doi:10.1002/(SICI)1097-0347(199710)19:7<620::AID-H ED10>3.0.CO;2-6

[8] C. Y. Hsing, Y. K. Wong, C. P. Wang, C. C. Wang, R. S. Jiang, F. J. Chen and S. A. Liu, "Comparison between Free Flap and Pectoralis Major Pedicled Flap for Reconstruction in Oral Cavity Cancer Patients-A Quality of Life Analysis,” Oral Oncology, Vol. 47, No. 6, 2011, pp. 522-527. doi:10.1016/j.oraloncology.2011.03.024

[9] D. B. Chepeha, G. Annich, M. A. Pynnonen, J. Beck, G. T. Wolf, T. N. Teknos, C. R. Bradford, W. R. Carroll and R. M. Esclamado, "Pectoralis Major Myocutaneous Flap vs Revascularized Free Tissue Transfer: Complications, Gastrostomy Tube Dependence, and Hospitalization," Archives of Otolaryngology—Head \& Neck Surgery, Vol. 130, No. 2, 2004, pp. 181-186. doi:10.1001/archotol.130.2.181

[10] D. Novakovic, R. S. Patel, D. P. Goldstein and P. J. Gullane, "Salvage of Failed Free Flaps Used in Head and Neck Reconstruction,” Head \& Neck Oncology, Vol. 1, 2009, p. 33. doi:10.1186/1758-3284-1-33

[11] A. Talesnik, B. Markowitz, T. Calcaterra, C. Ahn and W. Shaw, "Cost and Outcome of Osteocutaneous Free-Tissue Transfer versus Pedicled Soft-Tissue Reconstruction for Composite Mandibular Defects,” Plastic and Reconstructive Surgery, Vol. 97, No. 6, 1996, pp. 1167-1178. doi:10.1097/00006534-199605000-00011

[12] H. H. El-Marakby, "The Reliability of Pectoralis Major Myocutaneous Flap in Head and Neck Reconstruction,” Journal of Egyptian National Cancer Institute, Vol. 18, No. 1, 2006, pp. 41-50.

[13] J. N. McLean, G. W. Carlson and A. Losken, "The Pectoralis Major Myocutaneous Flap Revisited: A Reliable Technique for Head and Neck Reconstruction,” Annals of Plastic Surgery, Vol. 64, No. 5, 2010, pp. 570-573.

[14] D. S. Schneider, V. Wu and M. K. Wax, "Indications for Pedicled Pectoralis Major Flap in a Free Tissue Transfer Practice,” Head \& Neck, Vol. 34, No. 8, 2012, pp. 11061110. doi:10.1002/hed.21868

[15] F. R. Pinto, C. R. Malena, C. M. Vanni, A. Capelli Fde, L. L. Matos and J. L. Kanda, "Pectoralis Major Myocutaneous Flaps for Head and Neck Reconstruction: Factors Influencing Occurrences of Complications and the Final Outcome," Sao Paulo Medical Journal, Vol. 128, No. 6, 2010, pp. 336-341. 
doi:10.1590/S1516-31802010000600005

[16] R. I. Zbar, G. F. Funk, T. M. McCulloch, S. M. Graham and H. T. Hoffman, "Pectoralis Major Myofascial Flap: A Valuable Tool in Contemporary Head and Neck Reconstruction,” Head \& Neck, Vol. 19, No. 5, 1997, pp. 412418.

doi:10.1002/(SICI)1097-0347(199708)19:5<412::AID-H ED8>3.0.CO;2-2

[17] M. L. Shindo, P. D. Costantino, C. D. Friedman, H. J. Pelzer, G. A. Sisson Sr., and F. J. Bressler, "The Pectoralis Major Myofascial Flap for Intraoral and Pharyngeal Reconstruction," Archives of Otolaryngology-Head \& Neck Surgery, Vol. 118, No. 7, 1992, pp. 707-711. doi:10.1001/archotol.1992.01880070037007

[18] J. P. Shah, V. Haribhakti, T. R. Loree and P. Sutaria, "Complications of the Pectoralis Major Myocutaneous Flap in Head and Neck Reconstruction," The American Journal of Surgery, Vol. 160, No. 4, 1990, pp. 352-355. doi:10.1016/S0002-9610(05)80541-0

[19] P. D. Righi, E. C. Weisberger, S. R. Slakes, J. L. Wilson, K. A. Kesler and P. B. Yaw, "The Pectoralis Major Myofascial Flap: Clinical Applications in Head and Neck Reconstruction,” American Journal of Otolaryngology, Vol. 19, No. 2, 1998, pp. 96-101. doi:10.1016/S0196-0709(98)90102-8 